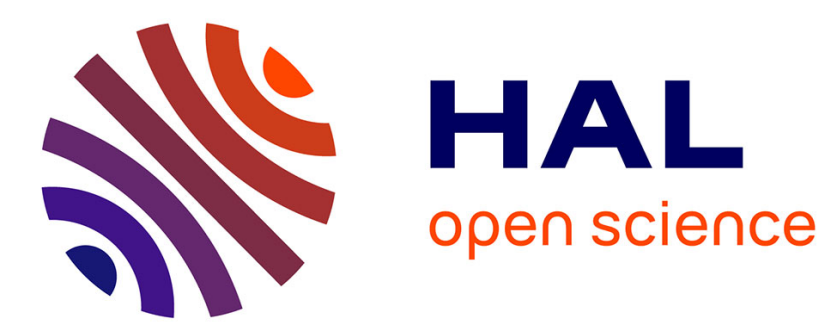

\title{
La Gelinotte et le noisetier en Ardenne belge
}

J.-P. Ledant

\section{To cite this version:}

J.-P. Ledant. La Gelinotte et le noisetier en Ardenne belge. Revue forestière française, 1995, 67 (3), pp.263-272. 10.4267/2042/26655 . hal-03444479

\section{HAL Id: hal-03444479 \\ https://hal.science/hal-03444479}

Submitted on 23 Nov 2021

HAL is a multi-disciplinary open access archive for the deposit and dissemination of scientific research documents, whether they are published or not. The documents may come from teaching and research institutions in France or abroad, or from public or private research centers.
L'archive ouverte pluridisciplinaire HAL, est destinée au dépôt et à la diffusion de documents scientifiques de niveau recherche, publiés ou non, émanant des établissements d'enseignement et de recherche français ou étrangers, des laboratoires publics ou privés. 


\title{
LA GELINOTTE ET LE NOISETIER EN ARDENNE BELGE
}

\author{
J.-P. LEDANT
}

Les taillis furent longtemps l'habitat principal de la Gelinotte des bois (Bonasa bonasia) en Ardenne, et leur régression y est concomitante d'un profond déclin de l'espèce. Cependant, les situations connues en d'autres régions, ainsi que celles qui semblent avoir précédé la généralisation du régime du taillis en Ardenne, ont permis de douter du caractère inconditionnel du lien entre la Gelinotte et ces peuplements forestiers. C'est pourquoi l'on a cherché à préciser davantage les exigences d'habitat de l'espèce en vue de définir d'éventuelles mesures de protection plus réalistes que la restauration à grande échelle de ce régime forestier désuet. Dans le cadre de ces recherches, l'on a trouvé, qu'outre les petites surfaces de boulaies pubescentes et aulnaies oligotrophes, la Gelinotte habite en Ardenne belge des peuplements à sous-étage de Noisetiers (Corylus avellana) et qu'en dépit de l'ancienne dénomination de poule des coudriers, ce lien est nouveau ou du moins s'est renforcé dans les dernières décennies. Le but de cet article est de montrer comment ce résultat a été obtenu, quelles interprétations en sont données, et quelles conclusions en sont tirées pour une sylviculture compatible avec la conservation de la Gelinotte.

\section{LE LIEN ENTRE LA GELINOTTE ET LE NOISETIER}

\section{Méthodes}

Plus d'un millier de données d'observation de Gelinottes (y compris les observations d'indices de présence) ont été rassemblées par recherches bibliographiques et par enquêtes auprès des forestiers, des chasseurs et des naturalistes dans l'Ardenne belge. Chaque fois que possible les données ont été réparties par catégorie de peuplement, définie selon la classification des statistiques forestières qui en donnent les surfaces. Des densités relatives ont ainsi pu être calculées, qui confirmèrent et quantifièrent le lien de la Gelinotte avec les taillis, taillis-sous-futaie et pineraies sur taillis (Ledant et Devillers, 1991). Au sein de cet ensemble, dont les catégories montrent des densités du même ordre de grandeur et près de 15 fois supérieures à celle des autres peuplements, une analyse de la distribution spatio-temporelle des observations (Ledant, 1995) a montré que la probabilité de détecter l'espèce n'est pas homogène et qu'il pouvait donc être envisagé d'y préciser davantage la sélection d'habitats, selon des critères qui traversent ceux de la classification sylvicole suivie dans ces statistiques forestières.

Des relevés de terrain ont donc été effectués, de manière à comparer des sites d'observation à un échantillon aléatoire de sites sans observation, au sein des taillis, simples et composés, considérés au sens large ( $y$ compris des peuplements en état avancé de conversion, mais à souille ligneuse). En raison de l'étalement des données dans le temps et du souci de mettre en relation les évolutions des milieux et des effectifs, l'on a distingué dans ces comparaisons les sites d'observation récente 


\section{J.-P. LEDANT}

de ceux d'observation plus ancienne. Seules les données les plus fiables et les plus précises ont été utilisées à ces fins, à l'exclusion des observations faites durant la période d'erratisme postnuptial.

La comparaison a porté sur les paramètres jugés potentiellement déterminants : la structure et la composition des strates ligneuses inférieures (densité de souches, nombre de brins par souche, répartition par espèce, inventaire des brins par classe de grosseur) et la composition botanique du sous-bois (relevé phytosociologique). Les parcelles échantillonnées sont d'une dizaine d'ares en moyenne, selon l'hétérogénéité constatée de la végétation. D'une façon générale, elles sont représentatives de la parcelle sylvicole concernée.

Trois groupes de stations ( $\mathrm{A}, \mathrm{B}, \mathrm{C})$ ont ainsi été comparés:

- Le groupe A rassemble les 16 stations où des observations postérieures à 1980, effectuées en dehors de la période présumée d'erratisme postnuptial (septembre à novembre), sont connues avec une relativement grande précision de localisation (point sur carte au 1/25 000).

- L'ensemble B est un échantillon de 19 sites d'observations (sauf erratisme postnuptial) effectuées dans les années 1970-1979, à au moins $1 \mathrm{~km}$ de toute observation plus récente qui soit connue. Du fait de l'âge de la donnée, la précision de localisation est en général inférieure à celle du groupe A. À défaut de connaître le point exact d'observation, le relevé est effectué en un point représentatif de l'aspect dominant des peuplements présents.

- L'ensemble $C$ regroupe 18 relevés situés à au moins $1 \mathrm{~km}$ de tout lieu d'observation connu, effectués en des points représentatifs de l'aspect dominant des peuplements présents.

Dans chaque cas, la précision de localisation semble acceptable du fait que les parcelles forestières montrent une grande homogénéité interne.

Le premier groupe (A) est à considérer comme étant en moyenne le plus proche d'un état favorable à la Gelinotte ou à son observation et le dernier le plus éloigné (C). Le groupe $B$, qui se distingue du groupe A par la date de la dernière observation connue, est en moyenne moins favorable que lui, depuis la dernière décennie au moins.

\section{Résultats}

Le tableau I (p. 265) résume les différences les plus importantes. Dans les peuplements les plus favorables à l'observation de Gelinottes $(A>B>C$ ), la biomasse ligneuse (surface terrière) est partagée en un nombre plus grand de tiges, une différence de structure due pour l'essentiel au Noisetier, Corylus avellana.

Les autres plantes caractéristiques de ces stations sont principalement les Ronces, Rubus fruticosus s.l., et l'Anémone, Anemone nemorosa, qui est toutefois sous-représentée dans nos relevés pour des raisons phénologiques. Du point de vue phytosociologique, les stations les plus favorables se rangent pour la plupart dans le Luzulo-Quercetum coryletosum (au sens de Noirfalise, 1984) ou dans le Stellario-Carpinetum.

En revanche la Myrtille, Vaccinium myrtillus, ainsi que les autres plantes de son groupe écologique (acidiphile), est relativement rare dans les meilleures stations à Gelinottes. Les taillis les plus défavorables à cet oiseau appartiennent au Luzulo-Quercetum typicum ou à d'autres variantes de sols acides, plus rarement au Carpinion sous des faciès à forte dominance de Charme.

Aucune corrélation positive n'est détectée entre la présence de la Gelinotte et celle d'autres essences ligneuses que le Coudrier, même réputées favorables (Hêtre, Sorbier, Charme, Épicéa...), ce qui suggère que leur présence n'est pas limitante dans les conditions de l'Ardenne belge (1).

(1) Il en va de même d'autres facteurs du milieu souvent jugés favorables, comme la proximité des cours d'eau (Ledant et Devillers, 1991). 
Tableau la

Comparaison des trois types de stations:

A (sites d'observation récente), B (sites d'observations plus anciennes), C (sites sans observation connue)

\begin{tabular}{|c|c|c|c|}
\hline & A & $\mathrm{B}$ & $\mathrm{C}$ \\
\hline Densité de tiges ligneuses $(1000 / \mathrm{ha}) \ldots \ldots \ldots \ldots$ & 10,3 & 4,7 & 4,5 \\
\hline Densité de tiges de Noisetier $\ldots \ldots \ldots \ldots \ldots \ldots$ & 7,6 & 2,2 & 1,6 \\
\hline Proportion de Noisetiers (\%) $\ldots \ldots \ldots \ldots \ldots$ & 14,2 & 20,3 & 31,2 \\
\hline 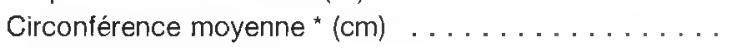 & 12,3 & 15,1 & 17,2 \\
\hline Surface terrière $\left(\mathrm{m}^{2} / \mathrm{ha}\right) \ldots \ldots \ldots \ldots \ldots$ & 94 & 79 & 55 \\
\hline Recouvrement moyen des Ronces $(\%) \ldots \ldots \ldots \ldots$ & 34 & 14 & 6 \\
\hline Fréquence de la Myrtille (\%) . . . . . . . . . . . & 25 & 53 & 55 \\
\hline Recouvrement moyen de la Myrtille...$\ldots \ldots \ldots \ldots$ & 1 & 6 & 16 \\
\hline
\end{tabular}

* Au sens dendrométrique : circontérence réalisant la surface terrière moyenne.

Tableau Ib

Signitication statistique des différences

\begin{tabular}{|c|c|c|c|}
\hline & $A-B$ & $B-C$ & $A-C$ \\
\hline Densité de tiges ligneuses $\ldots \ldots \ldots \ldots \ldots \ldots$ & * & - & * \\
\hline Densité de tiges de Noisetier $\ldots \ldots \ldots \ldots \ldots$ & * & - & * \\
\hline Circonférence moyenne $\ldots \ldots \ldots$ & - & - & * \\
\hline Surface terrière $\ldots \ldots \ldots \ldots \ldots \ldots \ldots \ldots$ & - & - & - \\
\hline Recouvrement moyen des Ronces $\ldots \ldots \ldots \ldots \ldots$ & - & - & * \\
\hline Recouvrement moyen de la Myrtille . . . . . . . . . . & * & * & * \\
\hline
\end{tabular}

Des différences A-B, B-C et A-C, celles marquées ci-dessus d'une astérisque ( $\left(^{\star}\right)$ sont statistiquement significatives au niveau $0,05 \%$ selon le test $t$ de différence de moyennes. Celles marquées d'un tiret s'avèrent non significatives selon ce test. Toutetois, le fait que tous les chitfres de B sont intermédiaires entre ceux de A et ceux de C suggère que les ditférences observées ne sont par purement aléatoires.

\section{Interprétation}

La date de dernière observation est considérée comme un indicateur de la probabilité d'observer la Gelinotte, qui s'avère donc corrélée à l'abondance du Noisetier.

Il reste à départager les influences sur ce résultat de la présence objective de l'oiseau d'une part et de la probabilité d'y repérer un oiseau présent d'autre part, qui est liée à la présence des observateurs potentiels et au couvert. Mais la fréquentation par les observateurs potentiels ne semble pas avoir contribué aux résultats obtenus :

- la plupart des observations ont été etfectuées le long de chemins de passage, ce qui les rend relativement indépendantes d'un lien éventuel entre l'habitat et la présence des observateurs potentiels ;

- la part des observations dues à des forestiers, donc aux personnes les plus susceptibles de s'attarder davantage dans un type de peuplement que dans un autre, est taible et du même ordre de grandeur $(20 \%)$ dans chacune des catégories.

La détectabilité n'intervient pas non plus, car la visibilité est au contraire la plus taible dans les sousbois denses de Noisetier, où la Gelinotte a pourtant été le plus observée. La conclusion est donc tirée que les taillis riches en Noisetier sont les plus tavorables à la présence objective de la Gelinotte en Ardenne belge. 
Les explications possibles à cette préférence d'habitat sont les suivantes :

- la Gelinotte est attirée par les peuplements à structure multicaule, de type taillis, et le Noisetier est l'essence qui contribue le plus à une telle structure ;

- la structure spécifique du Noisetier est particulièrement favorable, grâce à l'encombrement de la cime (rôle d'abri) et aux branches arquées, qui lui permettent de s'y tenir et de s'y déplacer aisément ; la Gelinotte les utilise sans doute pour atteindre les chatons et pour s'y reposer ; le Noisetier est en effet un perchoir préférentiel pour la nuit (Schmidt, 1991), peut-être parce que l'élasticité de ses branches favorisent la détection des prédateurs (Chat, Felis sylvestris; Marte, Martes martes) qui y grimperaient (Heim de Balsac, 1935);

- la Gelinotte se nourrit abondamment des chatons de Noisetier en hiver, du moins aux basses altitudes en Europe centrale et occidentale (Bergmann et al., 1982 ; Dronneau, 1984 ; Jacob, 1988a ; Lieser, 1986 ; Schatt, 1991b, 1993) ;

- les Ronces (essentiellement celles du groupe Rubus fruticosus) semblent également jouer un grand rôle alimentaire dans nos régions, d'après les études géographiquement les plus proches (Heim de Balsac, 1935 ; Lieser, 1986 ; Schatt, 1991b) ;

- l'Anémone des bois, Anemone nemorosa, qui est la fleur la plus abondante (après la floraison du Noisetier) dans les taillis considérés du Luzulo-Quercetum coryletosum, entre pour une part essentielle dans l'alimentation printanière selon les études biélorusses (Gavrin, 1969 in Dronneau, 1984) et suédoises (Ahnlund et Helander, 1975), et de même Jacob (1988b) constate l'importance des fleurs (en général) dans le régime alimentaire de la Gelinotte au printemps.

Les stations, souvent acidiphiles, pauvres en Noisetier et en Gelinottes, présentent des ressources hivernales relativement analogues à celles que la Gelinotte exploite par exemple dans les Vosges du Nord (Dronneau, 1982, in Dronneau, 1984) et les étages supérieurs du Jura (Zbinden, 1979 ; Jacob, 1988b; Schatt, 1991b, 1993). Bien qu'il y existe moins de fleurs que dans les peuplements à Noisetier, il semble que ce soit donc surtout la structure qui ne convienne pas, par manque d'une strate arbustive (faible densité de tiges et forte grosseur moyenne au tableau I, p. 265).

\section{LES TENDANCES DU LIEN ENTRE LA GELINOTTE ET LE NOISETIER}

\section{Méthodes}

Les surfaces de "taillis", au sens large du terme (taillis simples ou composés, en conversion plus ou moins avancée), étant divisées en mailles de 100 ha, celles-ci ont été classées selon deux critères :

- la date de la dernière observation connue,

- le principal critère discriminant identifié ci-dessus, à savoir la densité de Noisetiers.

Trois classes ont été distinguées pour chacun de ces deux critères:

- $\mathrm{Pa}, \mathrm{Pb}, \mathrm{Pc}$ : mailles avec observation postérieure à 1980, avec observation comprise entre 1970 et 1979, sans observation postérieure à 1970 (à ce niveau sont prises en compte toutes les observations, y compris celles qui, localisées avec trop peu de précision, n'ont pas donné lieu aux relevés de végétation considérés plus haut)

- P, I, R : mailles pauvres, intermédiaires ou riches en Noisetier (0 tige/are, 1-36 tiges/are, plus de 36 tiges/are).

On ne connaît pas :

- l'effectif de Pc,

- l'effectif de R, de I et de P.

On peut connaître en revanche : 
- les effectifs de $\mathrm{Pa}$ et $\mathrm{Pb}$, car ils se déduisent de l'inventaire des données d'observations,

- la proportion de mailles de type $\mathrm{R}, \mathrm{I}$ et $\mathrm{P}$ dans chacune des classes $\mathrm{Pa}, \mathrm{Pb}$ et $\mathrm{Pc}$, puisque les relevés de végétation $(A, B, C)$ ont été effectués dans des échantillons considérés comme représentatits de ces classes: pour les deux premières il s'agit des sites d'observation connus avec le plus de précision et, pour la dernière, d'échantillons purement aléatoires.

Il est donc possible de comparer, à une constante près, et séparément pour chaque catégorie $\mathrm{R}, \mathrm{I}$, $P$, le nombre (relatif) de mailles où la Gelinotte fut observée après 1980 et le nombre de mailles où elle le fut après 1970. Le rapport entre ces deux nombres étant une mesure de la vitesse de déclin, il est également possible de comparer la vitesse de déclin entre les catégories $R$, I et $P$.

\section{Résultats}

Le tableau II (ci-dessous) donne la répartition des relevés de végétation selon la date de dernière observation de Gelinotte et la richesse en Noisetier.

L'inventaire des données, y compris celles trop imprécises pour avoir été utilisées plus haut, indique que des Gelinottes ont été récemment (après 1980) détectées dans, à une petite imprécision près, quelque 37 mailles et plus anciennement (entre 1970 et 1980) dans quelque 46 mailles; donc nous considérons $\mathrm{Pa}=37$ et $\mathrm{Pb}=46$.

Tableau II

Distribution des nombres de relevés, selon la date de dernière observation connue et la richesse en Noisetier

\begin{tabular}{|c|c|c|c|}
\hline & Groupe A & Groupe B & Groupe C \\
\hline R : Riche $\ldots \ldots \ldots \ldots$ & 9 & 5 & 4 \\
\hline I : Intermédiaire $\ldots \ldots \ldots$. & 6 & 9 & 3 \\
\hline P: Pauvre $\ldots \ldots \ldots \ldots$ & 1 & 5 & 11 \\
\hline
\end{tabular}

Tableau III

Distribution du nambre de mailles d'après la date de dernière observation connue de la Gelinotte et la richesse en Noisetier

\begin{tabular}{|l|c|c|c|c|}
\hline & Pa & Pb & Pc & Total \\
\hline Riche . . . . . . . . . . . . . . . . . & 21 & 12 & $x-33$ & $x$ \\
Intermédiaire . & 14 & 22 & $y-36$ & $\mathrm{z}$ \\
Pauvre . . & 37 & 12 & $z-14$ & \\
Total . . & 46 & & \\
\hline
\end{tabular}

La distribution estimée du nombre de carrés selon la richesse en Noisetier se déduit comme indiqué au tableau III (ci-dessus), en reprenant les mêmes proportions entre les catégories $R$, I et $P$ qu'au tableau II (ci-dessus).

On constate (tableau IV, p. 268) que :

- Le rapport (1)/(2) entre les chiffres des deux premières colonnes diminue de $R$ vers $P$, ce qui indique une vitesse de diminution croissante de $\mathrm{R}$ vers $\mathrm{P}$. La diminution a donc été la plus forte dans les milieux les plus pauvres en Noisetier.

- Dans l'hypothèse d'une stabilité, on s'attend à ce que le rapport (1)/(2) soit compris entre 0,5 et 1 . 


\begin{tabular}{|l|c|c|c|}
\hline & $1980-89(1)$ & $1970-79(2)$ & $(1) /(2)$ \\
\hline R: Riche $\ldots \ldots \ldots \ldots \ldots \ldots$ & $21 / x$ & $33 / x$ & 0,64 \\
I : Intermédiaire $\ldots \ldots \ldots \ldots$ & $14 / \mathrm{y} \ldots \ldots \ldots$ & $36 / \mathrm{y}$ & 0,39 \\
P: Pauvre $\ldots \ldots \ldots \ldots \ldots$ & $2 / \mathrm{z}$ & $14 / \mathrm{z}$ & 0,14 \\
\hline
\end{tabular}

En effet, la recherche de points de présence sur 20 ans (1970-1989) devrait fournir plus de points que la même recherche sur 10 ans mais moins du double, en raison du plafonnement inévitable de la courbe liant le nombre de sites trouvés et la durée de la recherche. Donc ces chiffres indiquent une diminution pour les milieux $I$ et $P$, et ne disent rien de l'éventuelle évolution dans $R$, où les résultats obtenus sont ceux correspondant à une stabilité, ou en tout cas à des changements modérés.

- La densité semble avoir été plus grande en 1970-1979 dans les milieux classés aujourd'hui comme I que dans ceux classés comme R. En effet, pour que la densité des années 1970-1989 dans $R(=33 / x)$ soit supérieure à celle dans I $(=36 / y)$, il faut mathématiquement que y soit supérieur à 1,09 x. Or la proportion $4 / 3$ entre les nombres de stations $R$ et I dans le groupe $C$ (tableau II, p. 267) est un estimateur de $x-33 / y-36$, de sorte que cette condition n'est remplie que si $y$ est de loin inférieur à 34. Or (tableau III, p. 267), y est nécessairement supérieur ou égal à 36.

\section{Interprétation et commentaires}

Pour expliquer le déclin le plus rapide dans les milieux pauvres en Noisetier, cinq causes théoriques possibles peuvent être envisagées:

- L'abandon préférentiel des milieux marginaux, résultant d'une diminution due à des facteurs autres qu'un changement de l'habitat ; cette explication ne suffit pas puisqu'il semble que I ait été un meilleur habitat que $R$.

- Une modification de la sélection d'habitats, en l'occurrence un déplacement de préférences, des milieux intermédiaires I vers des milieux plus riches en Noisetier $\mathrm{R}$ ou plus fermés; ce changement trouverait une explication dans une augmentation de la pression de prédation: l'Autour (Accipiter gentilis), qui est un prédateur important de la Gelinotte (Bergmann et al., 1982 et divers témoignages locaux), a effectivement fort réaugmenté à partir de la décennie 1970 (Ledant et al., 1983 ; Clotuche et al., 1988).

- Des changements périodiques de l'habitat, liés aux cycles forestiers : selon cette explication seul un décalage chronologique distinguerait les peuplements où la Gelinotte fut observèe dans les années 1970, à faible densité actuelle de Noisetier, de ceux où la Gelinotte fut observée plus récemment, à forte densité actuelle de Noisetier. Ceci suppose :

- un sous-bois comparable quant aux espèces indicatrices des conditions pédologiques,

- une périodicité de coupes de l'ordre de 20 ans, puisque un demi-cycle séparerait les deux groupes,

- des densités d'observations dans I en 1970-1979 du même ordre de grandeur que celles dans R en 1980-1989,

- une distribution d'allure gaussienne (en cloche), et non pas hyperbolique ou parabolique, du nombre de tiges de Noisetier classées par classe de grosseur dans chacune des catégories,

- des tiges en moyenne plus grosses là où elles sont le moins nombreuses (peuplements plus vieux),

- un changement continu de l'habitat par raréfaction du Noisetier,

- un changement continu de l'habitat par la modification d'autres paramètres que l'abondance du Noisetier.

Or, aucune de ces conditions n'est remplie. Cette explication est donc rejetée. 
- Une modificafion continue de l'habitat par raréfaction du Noisetier: cette hypothèse semble pouvoir être retenue comme explication partielle et insuffisante. Dans les chênaies acidiphiles, le Noisetier aurait connu une phase pionnière après l'abandon de l'essartage (Noirfalise et Sougnez, 1957 ; Noirfalise et Thill, 1958, 1960) et la ségrégation de strates qui s'est produite avec le vieillissement et la conversion des taillis en futaie sur souche : on s'attend alors logiquement à une raréfaction du Noisetier après cette phase pionnière, d'autant plus que le grand gibier a fort augmenté. La raréfaction préférentielle du Noisetier sur les sols les plus pauvres découlerait de ce que le déclin de l'essartage y aurait été le plus précoce, pour des raisons économiques évidentes, et de ce que l'impact du gibier sur ces stations à faible productivité est le plus lourd. Toutefois, l'explication est insuffisante parce qu'elle ne rend pas compte de ce que les densités de Gelinotte étaient plus fortes dans les années 1970 dans les milieux I (actuellement peu fournis en Noisetiers) qu'actuellement dans les milieux R (riches en Noisetier), car il est invraisemblable que ces milieux I fussent dans les années 1970 plus riches en Noisetier que R dans les années 1980.

- Une altération de l'habitat sous d'autres aspects que la dynamique du Noisetier, ce qui suppose que dans les stations pauvres en Noisetier, donc aussi en Ronces et Anémone, la Gelinotte dépendait autrefois d'autres ressources, dont elle ne disposerait plus. Jeunes, les taillis présentaient de fait, indépendamment de leur composition, les traits structuraux identifiés comme favorables à la Gelinotte : le nombre de tiges par hectare et celui par cépée étaient nécessairement grands, et corrélativement ces tiges jeunes étaient en moyenne de faible grosseur. Parallèlement, les ressources alimentaires étaient présentes : les stations les plus acidiphiles, pauvres en Noisetier, étaient notamment pourvues de Sorbiers, Bouleaux et Myrtille. Maintenant que les taillis ne sont plus exploités et ont vieilli, la structure multicaule du taillis s'est perdue : le nombre de tiges a diminué, de manière spontanée ou artificielle, et les tiges qui ont survécu se sont épaissies. En même temps, les plantes d'inférêt alimentaire se sont raréfiées sous la pression du grand gibier ou, conséquence du changement structural, sont devenues moins disponibles, les espèces arborescentes ayant poussé en hauteur (Bouleaux, Sorbier).

Cette cinquième hypothèse, qui se réfère principalement à des changements de végétation évidents, en particulier le vieillissement des taillis, n'est pas incompatible avec celle d'une raréfaction spécifique du Coudrier. Les stations où les dernières observations datent des années 1970 auraient pour la plupart été des taillis vieillissants, les derniers à pouvoir encore abriter des Gelinottes, capacité qu'ils auraient pour un temps conservée à la faveur d'un maigre peuplement de Noisetier, et qu'ils auraient perdue la décennie suivante. À ce niveau, un problème vient toujours de l'écart de vitesses entre la cause, l'évolution forestière plutôt lente, et la conséquence, la diminution rapide de la Gelinotte. Mais peut-être suffit-il que la structure des peuplements, qui évolue continûment, franchisse un seuil pour entraîner alors en quelques années un déclin rapide de la Gelinotte.

\section{Conclusion quant aux causes de diminution}

Depuis près d'un siècle, l'abandon du régime du taillis, associé à une charge croissante en gibier, a causé indubitablement une modification de la végétation, dans un sens défavorable à la Gelinotte, en même temps que la pression de prédation a changé.

Si la Gelinotte se maintient le mieux dans des taillis riches en Noisetier, c'est en grande partie parce que cette petite essence buissonnante est la seule à pouvoir encore constituer une strate arbustive dense en l'absence de recépages périodiques ou longtemps après leur cessation. Tout se passe comme si l'hypothèse suivante était vraie:

- En dehors des forêts édaphiques (forêts sur alluvions, tourbe, ravins, affleurements rocheux), la Gelinotte occupait en Ardenne l'ensemble des chênaies acidiphiles (Luzulo-Quercetum), ainsi que les chênaies-charmaies acidoclines du Stellario-Carpinetum, à l'époque où elles étaient encore traitées en taillis. Les taillis en recroissance, composés pour l'essentiel de Quercus petraea et Betula verru- 


\section{J.-P. LEDANT}

cosa, avec, dans les stations les plus riches (Luzulo-Quercetum coryletosum, Stellario-Carpinetum), une bonne réprésentation de Corylus avellana, assuraient les besoins structuraux. Sorbus aucuparia, Vaccinium myrtillus, Betula verrucosa, Betula pubescens et, dans les stations les plus riches, Corylus avellana fournissaient l'alimentation hivernale. Les espèces de coupes (Rubus sp., Rubus idaeus, Sambucus racemosa), ainsi que Frangula alnus, Sorbus aucuparia, Vaccinium myrtillus par exemple donnaient les fruits dont la Gelinotte se nourrit.

- Quand le régime du taillis fut abandonné, l'absence de recépage supprima la structure arbustive et buissonnante des essences principales (Chênes et Bouleaux principalement), qui vieillirent, et la structure évolua de façon divergente selon que le Noisetier, espèce ligneuse de petite taille et drageonnante, a disposé ou non de conditions de sol et d'éclairement qui lui conviennent. Dans le Luzulo-Quercetum coryletosum et le Stellario-Carpinetum, généralement sur les anciens sarts, le Noisetier a maintenu spontanément une strate arbustive favorable, où il a pu prendre un développement nouveau à la faveur de l'éviction des essences principales dans la même strate. Dans les sousassociations plus acidiphiles en revanche, comme le Luzulo-Quercetum typicum, ni le Noisetier ni aucun autre arbuste ne purent prendre le relais. Dans les associations plus basiphiles, c'est le couvert dense du Charme qui a limité le Noisetier. Dans certains cas, c'est l'ombrage du Hêtre, essence climacique qui tend à envahir les chênaies là où ses semenciers sont présents, qui élimine ou affaiblit le Noisetier. Enfin, partout mais surtout en stations marginales, l'impact du grand gibier, devenu plus lourd avec la croissance du cheptel et la réduction de ses propres ressources alimentaires, a aggravé cette situation.

- Dans ces stations où le Noisetier est rare, ces évolutions forestières relativement lentes ont produit une régression rapide de la Gelinotte, par la synergie entre la dégradation de structure de végétation, avec raréfaction éventuelle de Coudriers devenus limitants, et de la croissance de la pression de prédation, qui fragilise l'espèce dans les milieux les moins touffus.

\section{CONCLUSIONS : ORIENTATIONS DE GESTION}

La Gelinotte est devenue très rare en Ardenne belge. Impossible à dénombrer, elle ne compte peutêtre plus qu'une cinquantaine de couples, tandis qu'elle peut être considérée comme l'une des espèces prioritaires des efforts de conservation de la biodiversité en forêt wallonne (Ledant, 1993).

Les chênaies à sous-bois de Noisetier, et en conversion vers la futaie, offrent l'issue espérée à l'impasse que constituait la dépendance apparente de la Gelinotte en Ardenne envers le régime, révolu et à divers points de vue non souhaitable, du taillis. Elles constituent les principaux habitats qui restent disponibles à l'espèce. Leur conservation est donc recommandée, sur des surfaces suffisantes, complétées éventuellement par d'autres habitats favorables : taillis en exploitation maintenus localement ou à titre transitoire, forêts édaphiques (aulnaies, boulaies, forêts de ravins). Cet ensemble constitué en un réseau quasi continu demande une gestion cohérente et des mesures communes, telles que le contrôle du dérangement, du surpâturage par le gibier et de la prédation, en particulier celle causée par le sanglier en forte densité.

Les chênaies à Noisetier ne sont pas seulement à protéger contre leur destruction ou transformation, elles doivent encore être entretenues et régénérées, et de préférence améliorées.

En effet, la pérennité de ces forêts issues de la conversion de taillis autrefois essartés n'est malheureusement pas assurée, aussi bien quant au peuplement principal de Chênes qu'en ce qui concerne le sous-étage à dominance de Noisetier. Des efforts particuliers de régénération du Chêne, auquel d'autres essences de lumière peuvent être associées, sont requis, éventuellement par plantation artificielle et avec protection contre le gibier, sans quoi ces forêts évoluent souvent vers des 
peuplements denses et inhospitaliers de Hêtres, d'Épicéas ou de Douglas. Au vu de la raréfaction généralisée du bois de Chêne, une telle politique semble d'ailleurs défendable du point de vue économique, du moins dans une logique de gestion durable des ressources. Le traitement qui convient à la Gelinotte est celui qui favorise le sous-bois et les essences secondaires : futaie claire, à larges écartements précoces, et régénération accompagnée d'un bourrage. L'évolution du sous-bois de Noisetier est de même à surveiller en vue d'éventuelles interventions ultérieures, qui restent à définir.

Les améliorations possibles portent sur la structure et la composition du sous-étage. Plusieurs études étrangères suggèrent que la Gelinotte pourrait être favorisée par une plus grande hétérogénéité du sous-étage, où des plages ensoleillées, utiles à l'alimentation des jeunes et au confort thermique, jouxtent des cépées denses où les oiseaux puissent s'abriter : une telle structure peut être obtenue par un programme de recépages par souches individuelles ou par bouquets, ou par l'aménagement d'un réseau de layons. D'autre part, il faut promouvoir le développement des espẻces d'intérêt alimentaire (Sorbiers, Bouleaux, Ronces, Fruitiers...), en particulier l'Aubépine car nos relevés phénologiques montrent qu'elle est probablement importante pour assurer la soudure après la floraison et la disparition précoce des chatons de Noisetier. S'il a été trouvé que les dernières Gelinottes d'Ardenne belge se confinent principalement dans les chênaies à Noisetier, il ne faut en effet pas conclure pour autant que ces peuplements constituent, en leur état actuel, les meilleurs habitats possibles. On se référera à Forêt wallonne (1993) pour des précisions supplémentaires concernant la gestion forestière favorable à la Gelinotte en Ardenne.

\section{J.-P. LEDANT}

INSTITUT ROYAL

DES SCIENCES NATURELLES DE BELGIQUE

Rue Vautier, 29

B-1040 BRUXELLES

\section{BIBLIOGRAPHIE}

AHNLUND (H.), HELANDER (B.). - The food of the Hazel Grouse (Tetrastes bonasia) in Sweden. - Viltrevy, 9, 1975, pp. 221-240.

BERGMANN (H.H.), KLAUS (S.), MÜLLER (F.), WIESNER (J.). - Das Haselhuhn. - Wittenberg Lutherstadt : A. Ziemsen Verlag, 1982.

CLOTUCHE (E.) et al. - Autour, Accipiter gentilis. In: Atlas des oiseaux nicheurs de Belgique / P. Devillers, W. Roggeman, J. Tricot, P. del Marmol, C. Kerwijn, J.P. Jacob et A. Anselin Éd. . - Bruxelles : IRSNB, 1988.

DESBROSSES (R.). - La Gelinotte des bois en Côte-d'Or. - Le Jean-le-Blanc, 24, 1985, pp. 47-61.

DRONNEAU (C.). - L'Écologie de la Gélinotte des bois (Bonasa bonasia) dans l'Est de la France. Premiers résultats. - Université Paris VI, 1982 (Rapport de DEA d'Écologie).

DRONNEAU (C.). - La Gélinotte des bois (Bonasa bonasia L.). Synthèse bibliographique. — Bulletin mensuel de I'ONC, $n^{\circ} 76,1984$, pp. 33-41; $n^{\circ} 77,1984$, pp. 43-47; n 78, 1984, pp. 27-36.

GAVRIN (V.F.). - [Écologie de la Gelinotte en forêt de Bialowieja] (en Russe). - Gosud. Zapov. Ochotn. Choz. Belovezskaja Pusca Minsk, 3, 1969, pp. 146-172.

HEIM DE BALSAC (H.). - Remarques sur la distribution et l'écologie de la Gélinotte Bonasa bonasia rupestris Brehm dans l'Est de la France. - Alauda, 7, 1935, pp. 227-242.

JACOB (L.). - Le Régime alimentaire de la Gelinotte des bois (Bonasa bonasia L.) : synthèse bibliographique. Gibier Faune sauvage, 5, 1988a, pp. 95-111. 


\section{J.-P. LEDANT}

JACOB (L.). - Régime alimentaire du Grand Tétras (Tetrao urogallus L.) et de la Gélinotte des bois (Bonasa bonasia L.) dans le Jura. - Acta Oecologica, Oecologia Generalis, 9, 1988b, pp. 347-370.

LEDANT (J.-P.). - Biodiversité et gelinottes en forêt wallonne. - Forêt wallonne, 18, 1993, pp. 3-12.

LEDANT (J.-P.). - Contribution méthodologique à l'identification des habitats d'espèces à observations rares et dispersées. In : Documents de Travail IRSNB (à paraître), 1995.

LEDANT (J.-P.). - Évolutions forestières et tendances chez la Gelinotte des bois (Bonasa bonasia) en Ardenne. - Aves, 30, 1993, pp. 173-180.

LEDANT (J.-P.). - La Situation de la Gelinotte des bois (Bonasa bonasia) en Ardennes et dans les régions voisines. - Aves, 27, 1990, pp. 159-172.

LEDANT (J.-P.), DEVILLERS (P.). - La Gelinotte des Bois en Ardenne et dans les régions voisines. Habitats, distribution et perspectives de conservation. - Documents de travail de l'IRSNB, 65, 1991.

LIESER (M.). - Untersuchungen zur Verbreitung und Ökologie des Haselhuhns (Bonasa bonasia) an der Mosel. — Freiburg: DBV-Mitteilungen Rheinland-Pfalz, 1986/1.

NOIRFALISE (A.). - Forêts et stations forestières de Belgique. - Gembloux: Presses Agronomiques, 1984

NOIRFALISE (A.), SOUGNEZ (N.). — Les Chênaies de l'Ardenne verviètoise. — Pédologie, VI, 1957.

NOIRFALISE (A.), SOUGNEZ (N.). - Les Forêts riveraines de Belgique. - Bull. Jard. Bot de l'État, 30, 1961, pp. 199-288.

NOIRFALISE (A.), THILL (A.). - Les Chênaies de l'Ardenne centrale. - Bull. Inst. Agron. et Stat. Rech. Gembloux, 26,1958, pp. 362-376.

NOIRFALISE (A.), THILL (A.). - Les Taillis sartés de l'Ardenne. In : UICN. Septième réunion technique, Athènes, 11-19 septembre 1958. - Volume II. Conservation du Sol et de l'Eau, 1960, pp. 364-370.

RONDEUX (J.), LECOMTE (H.), FAGOT (J.), LAURENT (C.), TOUSSAINT (A.). - Quelques donnèes statistiques récentes sur la forêt wallonne. - Bulletin de la Société royale forestiẻre de Belgique, 93, 1986, pp. 1-22.

SCHATT (J.). - Étude du régime alimentaire de la Gélinotte des bois Bonasa bonasia dans l'Ain (Première partie). - Alauda, 59, 1991b, pp. 89-100.

SCHATT (J.). - Étude du régime alimentaire de la Gélinotte des bois Bonasa bonasia dans l'Ain (Deuxième partie). - Alauda, 61, 1993, pp. 19-34.

SCHATT (J.). - La Gelinotte des bois. Biologie, éco-éthologie, mesures conservatoires. - Revue forestière française, vol. XLIII, n5, 1991a, pp. 445-461.

SCHMIDT (R.). - Das Haselhuhn und sein Lebensraum. In : Die Lohhecken des Öslings und das Haselhuhn. Luxembourg : Fondation "Hëllef fir d'Natur", "Lëtzebuerger Natur- a Vulleschutzliga", Musée national d'Histoire naturelle, Association des Universitaires au Service des Eaux et Forêts, 1991.

SOUGNEZ (N.). - Les Chênaies silicicoles de Belgique. - Colloques phytosociologiques III, les torêts acidiphiles, Lille, 1974.

TANGHE (M.). - Recherches sur l'écosystème forêt. Série E : Forêts de Haute Belgique. Contribution $n^{\circ} 12$. La végétation forestière de la Semois ardennaise. Troisième partie : Les associations forestières stationnelles de plateau et de plaine. - Bulletin de I'institut royal des Sciences naturelles de Belgique, vol. $46, n^{\circ} 30,1970$ pp. 1-76.

ZBINDEN (N.). - Zur Ökologie des Haselhuhns Bonasa bonasia in den Buchenwäldern des Chasseral, Faltenjura. - Der Orn. Beob., 76, 1979, pp. 169-214. 